\title{
A laboratory-based study to identify and speciate non- tuberculous mycobacteria isolated from specimens submitted to a central tuberculosis laboratory from throughout KwaZulu-Natal Province, South Africa
}

\author{
L Sookan, MB BCh, FCPath (SA) Micro; Y M Coovadia, MB ChB, FCPath (SA) Micro \\ Department of Medical Microbiology, Nelson Mandela School of Medicine, College of Health Sciences, University of KwaZulu-Natal, \\ Durban, South Africa, and National Health Laboratory Service, KZN Academic Complex, KwaZulu-Natal
}

Corresponding author: L Sookan (lishasookan@hotmail.com)

\begin{abstract}
Background. Non-tuberculous mycobacteria (NTM) are important environmental pathogens capable of causing a spectrum of infection. The different species exhibit varied geographical prevalence worldwide. Identification of the infecting organism may be helpful in determining the clinical significance of the isolate.

Objective. To describe the spectrum of NTM isolated from clinical specimens received at the National Health Laboratory Service central tuberculosis laboratory in KwaZulu-Natal Province, South Africa.

Method. In a laboratory-based prospective study, 200 suspected NTM were randomly selected over a period of 1 year and identified to species level using a commercially available DNA strip assay (GenoType Mycobacterium, CM/AS; Hain Lifescience, Germany).

Results. Of the 200 suspected NTM, 133 (66.5\%) were confirmed to be NTM by the molecular test. The most frequently isolated NTM species were Mycobacterium intracellulare (45.9\%), M. avium subspecies (11.3\%), M. gordonae (6.0\%) and M. kansasii (4.5\%).

Conclusion. It is important for laboratories to document the local spectrum of NTM because of the geographical variation in the different NTM species isolated. Although molecular tests for identifying NTM are relatively expensive, they have the advantage of providing rapid and accurate identification of the various NTM species.

S Afr Med J 2014;104(11):766-788. DOI:10.7196/SAMJ.8017
\end{abstract}

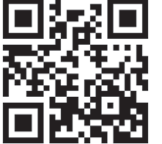

Non-tuberculous mycobacteria (NTM) is a term used to refer to species of the genus Mycobacterium that are not included in the Mycobacterium tuberculosis complex $(\mathrm{MTB})$. These organisms are ubiquitous in nature, and can be recovered from a wide array of environmental sources. ${ }^{[1]}$ NTM species are now increasingly being recognised as opportunistic pathogens capable of causing a spectrum of infections including pulmonary, lymphatic, skin and soft-tissue, and disseminated disease. ${ }^{[2]}$

More than 140 NTM species have been identified to date. Of these, 25 have been linked to human disease, exhibiting geographical variability with regard to prevalence of disease, species isolated and sites of infection. Identifying the NTM species, together with the site from which it was cultured, may be helpful in determining the clinical significance of the isolate. ${ }^{[3]}$

In the USA, M. avium complex was found to be the most common cause of NTM lung disease, followed by M. kansasii. ${ }^{[4]}$ In England and Wales, disease is most commonly caused by M. kansasii, and in Scotland by M. malmoense. ${ }^{[5]}$ In a locally based study, M. kansasii was the commonest isolate recovered from the sputum of miners. ${ }^{[6]}$ To the best of our knowledge, there are no data available in South Africa (SA) on the spectrum of NTM species recovered from all clinical specimens.

It is well recognised that clinicians have great difficulty in distinguishing between disease caused by NTM and MTBc. Although microscopy for acid-fast bacilli (AFB) enables rapid diagnosis of mycobacteria, it cannot differentiate $M$. tuberculosis from NTM. This requires culture, which may take up to 6 weeks. Speciation of NTM in a routine laboratory traditionally required a battery of biochemical tests and observation of growth rate and colony morphology. However, this is time consuming, error prone and labour intensive, and requires experienced personnel for interpretation of results. ${ }^{[7]}$ The introduction of molecular methods has led to a marked improvement in accuracy as well as turnaround time.

The GenoType Mycobacterium CM/AS (Hain Lifescience, Germany) is a commercial DNA strip assay based on reverse hybridisation of polymerase chain reaction products to their complementary probes. Although this molecular method is more expensive than the traditional methods, mycobacteria from positive liquid or solid cultures can be identified to species level rapidly and accurately.

The combination of the GenoType CM (for common mycobacteria) and Genotype AS (for additional species) allows for the detection of more than 40 clinically relevant mycobacteria species from cultured samples. ${ }^{[8]}$

\section{Methods}

This was a laboratory-based, prospective study to identify and speciate NTM isolated from specimens submitted to the National Health Laboratory Service (NHLS) central tuberculosis (TB) laboratory at Inkosi Albert Luthuli Central Hospital (IALCH) from patients throughout the province of KwaZulu-Natal Province (KZN), SA. This is the only laboratory performing mycobacterial culture on specimens received from public healthcare centres in KZN.

Ethical approval was obtained from the Biomedical Research Ethics Committee at the University of KwaZulu-Natal.

A retrospective analysis of laboratory records for the year 2009 revealed that the IALCH TB laboratory was isolating \pm 1200 suspected NTM and \pm 10000 MTBc annually from clinical specimens. In consultation with a statistician, it was agreed that 200 suspected 
NTM, which comprised $\pm 17 \%$ of the total number of NTM cultured annually, should be speciated to provide a statistically significant sample.

Random sampling for a period of 1 year was performed (January 2010 - December 2010). All specimens submitted for TB culture were processed according to the TB laboratory standard operating procedure. Samples included sputum, endotracheal aspirates, brochoalveolar lavage, sterile fluid, biopsy tissue, blood and cerebrospinal fluid. Isolates suspected to be NTM by colony morphology, biochemical tests (niacin and nitrate) and/or the SD Bioline TB Ag MPT64 Rapid Test were investigated further by a commercially available molecular test that speciates NTM. The GenoType Mycobacterium CM/AS was used to identify suspected NTM from liquid (BACTEC MGIT 960, Becton Dickinson, USA) or solid (Middlebrook 7H11; Becton Dickinson) culture media. DNA was extracted from the stored cultures according to the manufacturer's instructions. The GenoType assay was performed strictly according to the manufacturer's package insert.

The demographic characteristics of patients from whom the NTM was recovered were extracted from the NHLS TB laboratory database, and data analysis was performed using Microsoft Excel. Confidentiality of patient information was maintained throughout the study.

\section{Results}

Of a total of 200 suspected NTM cultures from 200 individual patients, $133(66.5 \%)$ were confirmed as NTM by the molecular assay. Of these 133 NTM, 114 were fully speciated by the molecular assay; 8 could not be speciated further as the molecular assays only contain probes that allow for the detection of 40 commonly isolated NTM, and the remaining 11 represented mixed NTM species. An additional 6 cultures were identified as $\mathrm{MTBc}$, and the remaining 61 cultures could not be speciated owing to contamination or an absence of mycobacterial DNA (Table 1).

Of the 133 NTM species, $M$. intracellulare $(n=61,45.9 \%)$, M. avium subspecies (ssp.) ( $n=15,11.3 \%), M$. gordonae $(n=8,6.0 \%)$ and M. kansasii $(n=6,4.5 \%)$ accounted for $90(67.7 \%)$ of isolates (Fig. 1).
All NTM were cultured from respiratory specimens, with the exception of an M. gordonae isolate that was cultured from a biopsy sample and an M. intracellulare isolate from a gastric washing.

Fifty-eight $(43.6 \%)$ of the NTM isolates were cultured from patients attending healthcare facilities in the eThekwini (Durban)

\begin{tabular}{ll} 
Table 1. Identification of suspected NTM & \\
\hline Organism & Isolates, $\boldsymbol{n}$ \\
\hline NTM & 133 \\
M. abscessus & 5 \\
M. asiaticum & 2 \\
M. avium ssp. & 15 \\
M. chelonae & 5 \\
M. fortuitum & 4 \\
M. gordonae & 8 \\
M. heckeshornense & 1 \\
M. interjectum & 2 \\
M. intracellulare & 61 \\
M. kansasii & 6 \\
M. lentiflavum & 1 \\
M. malmoense & 1 \\
M. scrofulaceum & 3 \\
NTM not speciated & 8 \\
Mixed NTM species & 11 \\
MTBc & 6 \\
Cultures with no mycobacterial DNA & 54 \\
Contaminated cultures & 7 \\
Total & 200 \\
NTM = non-tuberculous mycobacteria; M. = Mycobacterium; & \\
MTBc = . tuberculosis complex. & \\
\hline
\end{tabular}

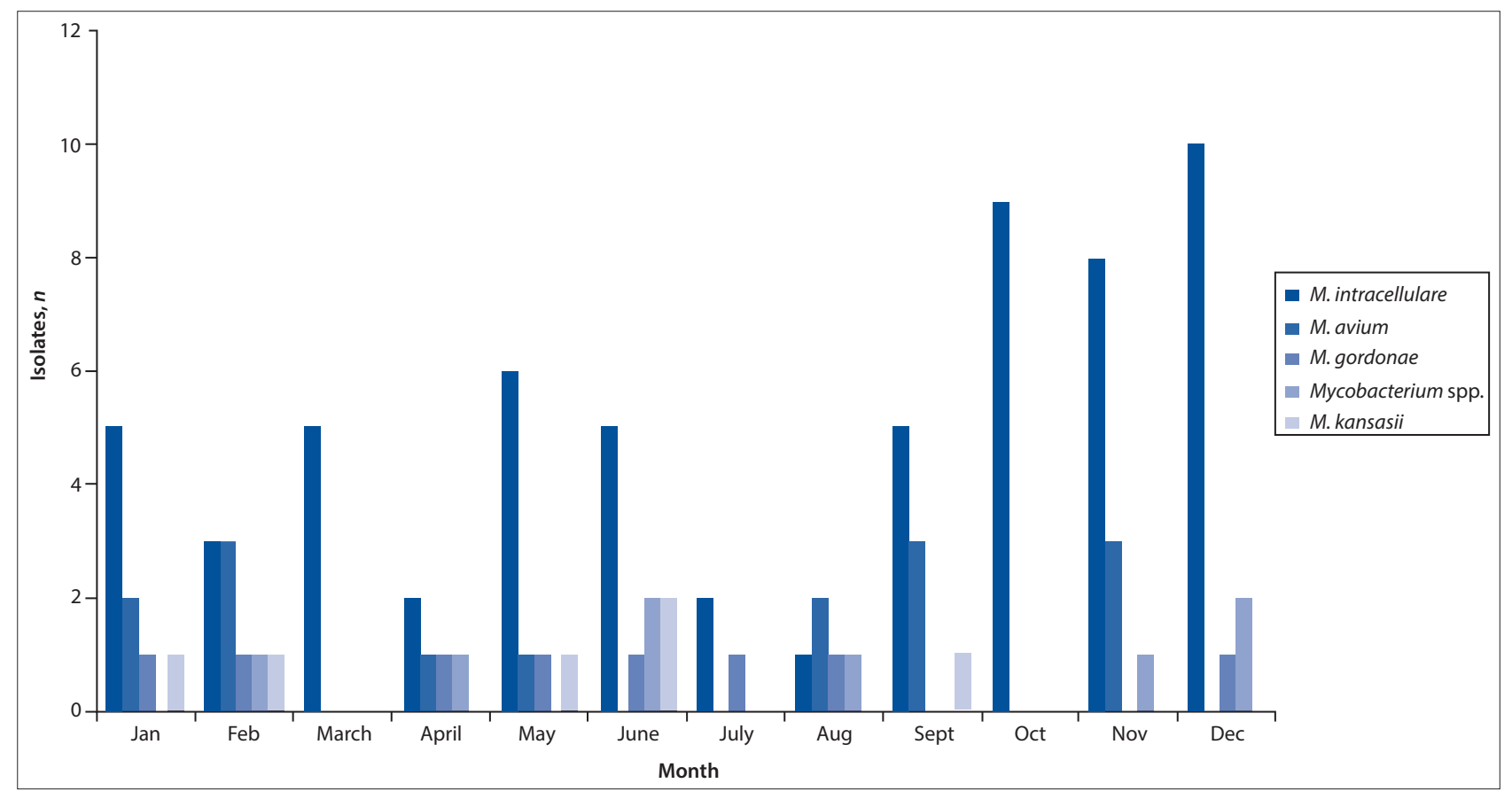

Fig. 1. Common Mycobacterium species isolated during the year 2010 
district. This was followed by $20(15.0 \%)$ and $11(8.3 \%)$ isolates from the Umkhanyakude and Uthungulu districts, respectively.

\section{Discussion}

In recent years there has been an increased awareness of NTM as disease-causing agents, especially in the growing HIV-infected population as well as in other immunosuppressed patients.

In countries with high prevalence rates of $M$. tuberculosis, patients in whom AFB are detected on microscopy or culture are generally assumed to have $\mathrm{TB}$ and treated as such. The incorrect diagnosis leads to unnecessary and/or incorrect treatment of many patients who are in fact colonised or infected with NTM. ${ }^{[9]}$

The most common species of NTM identified during the study period was $M$. intracellulare (45.9\%), followed by M. avium ssp. (11.3\%). These two species are often referred to as M. avium complex (MAC) and cannot be differentiated using phenotypic tests. There are, however, specific DNA probes in the molecular tests that allow for speciation of these two closely related and commonly isolated NTM. In this study, MAC accounted for 76 (57.1\%) of the 133 NTM isolates.

Six per cent of the cultured NTM were identified as M. gordonae. In the majority of cases this organism is considered to be nonpathogenic, and it is the most commonly isolated mycobacterial contaminant. ${ }^{[10]}$

M. kansasii, which is the most common agent causing NTM disease in England and Wales, made up only 4.5\% of NTM isolated in this study. Generally, tap water is reported to be the most frequent environmental source of this organism; infection may lead to lung disease that closely resembles tuberculosis. ${ }^{[10]}$

The spectrum and distribution of NTM in sub-Saharan Africa is not well known, as most data originate from the more developed nations in Northern Europe and North America. This may not necessarily be due to a low incidence of NTM disease in developing countries, but rather result from less priority being given to NTM in regions where the TB burden is overwhelming. Buijtels et al. ${ }^{[11]}$ reported that in Zambia, a country with a high prevalence of TB, MAC was the most frequently isolated NTM. Similarly, a study identifying NTM from clinical specimens in Ethiopia showed MAC to be the predominant species, followed by M. abscessus, M. gordonae, M. simiae and M. fortuitum. ${ }^{[12]}$ The high frequency of isolation of MAC in both studies is in keeping with our findings. In an SA study of NTM in miners, the two most common isolates were M. kansasii $(n=56)$ and M. scrofulaceum ( $n=14)$; only three of the isolates were identified as $M$. avium ssp. ${ }^{[6]}$ This is in contrast to our study, where MAC predominated, with $M$. kansasii making up only $4.5 \%$ of NTM recovered.

Ninety-three per cent of the NTM isolates were cultured from sputum. This is not surprising, as pulmonary TB remains the commonest form of TB in SA, hence the predominance of sputum samples being submitted for TB culture. Lung disease is also regarded as the most frequent clinical manifestation of NTM disease..$^{[10]}$

The province of KZN is divided geographically into 11 healthcare districts. Fifty-eight $(43.6 \%)$ of the NTM originated from patients presenting to healthcare facilities in the eThekwini (Durban) district. Twenty-one (36.2\%) of these isolates were identified as $M$. intracellulare. This organism was also found to be the predominant
NTM in all districts with the exception of Uthukela, where M. avium ssp. was the most common isolate. The high burden of TB disease in the eThekwini district, which has a large TB clinic as well as a TB referral hospital, may have resulted in a larger number of specimens being submitted for TB culture. This possibly accounted for the highest proportion of NTM being found in this district, rather than indicating a greater risk of NTM exposure.

\section{Study limitations}

This study had certain limitations. The absence of clinical and radiological data precluded determining the clinical significance of the NTM isolates. As a result, we could not determine whether disease was indeed due to a NTM, or if the organism was merely a contaminant. Furthermore, as a result of the long incubation period required for mycobacteria, many of the culture plates were found to be overgrown with non-mycobacterial contaminants, hindering the molecular process of identification.

\section{Conclusion}

The most common NTM isolated from patients with suspected mycobacterial disease were $M$. intracellulare and M. avium ssp., which is in keeping with findings elsewhere in the world. We believe that it is important for laboratories to document the local spectrum of NTM, even in developing countries, because of the geographical variation in the different NTM species isolated. Although the molecular tests for identifying NTM are relatively expensive and require expertise, they have the advantage of providing rapid and accurate identification of the various NTM species.

Acknowledgement. This research was conducted at the NHLS central tuberculosis laboratory, IALCH, KwaZulu-Natal, SA.

\section{References}

1. Falkinham JO III. The changing pattern of nontuberculous mycobacterial disease. Can J Infect Dis 2003;14(5):281-286 2. Chetchotisakd P, Kiertiburanakul S, Mootsikopun P, Assanasen S, Chaiwarith R, Anunnatsiri S.
Disseminated nontuberculous mycobacterial infection in patients who are not infected with HIV in Thailand. Clin Infect Dis 2007;45(4):421-427. [http://dx.doi.org/10.1086/520030]

3. Van Ingen J. Diagnosis of nontuberculous mycobacterial infections. Semin Respir Crit Care Med 2013;34(1):109-109. [http://dxdoi.org/10.1055/s-0033-1333569]

4. O'Brien RJ, Geiter LJ, Snider DE Jr. The epidemiology of nontuberculous mycobacterial diseases in the United States: Results from a national survey. Am Rev Respir Dis 1987;135(5):1007-1014.

5. British Thoracic Society. Management of opportunistic mycobacterial infections: Joint Tuberculosis Committee Guidelines 1999. Thorax 2000;55(3):210-218. [http://dx.doi.org/10.1136/thorax.55.3.210] 6. Corbett EL, Blumberg L, Churchyard GJ, et al. Nontuberculous mycobacteria: Defining disease in a prospective cohort of South African miners. Am J Respir Crit Care Med 1999;160(1):15-21. [http://
a dx.doi.org/10.1164/ajrccm.160.1.9812080]

7. Cook VJ, Turenne CY, Wolfe J, Pauls R, Kabani A. Conventional methods versus 16 S ribosomal Cook VJ, Turenne CY, Wolfe J, Pauls R, Kabani A. Conventional methods versus $16 \mathrm{~S}$ ribosomal
DNA sequencing for identification of nontuberculous mycobacteria: Cost analysis. J Clin Microbiol 2003;41(3):1010-1015. [http://dx.doi.org/10.1128/JCM.41.3.1010-1015.2003]

8. Hain Lifescience. GenoType Mycobacterial CM/AS. http://www.hain-lifescience.co.za/products microbiology/mycobacteria/genotype-mycobacterium-cmas.html (accessed 10 March 2010).

9. Jesudason MV, Gladstone P. Non tuberculous mycobacteria isolated from clinical specimens at a tertiary care hospital in South India. Indian J Med Microbiol 2005;23(3):172-175. [http://dx.doi. org/ $10.4103 / 0255-0857.1658$

10. Griffith DE, Aksamit T, Brown-Elliot BA, et al. An official ATS/IDSA statement: Diagnosis, treatment, and prevention of nontuberculous mycobacterial diseases. Am J Respir Crit Care Med 2007;175(4):367416. [http://dx.doi.org/ 10.1164/rccm.200604-571ST]

Buijtels PCAM, van der Sande MAB, de Graaff CS, et al. Nontuberculous mycobacteria, Zambia. Emerg Infect Dis 2009;15(2):242-249. [http://dx.doi.org/10.3201/eid1502.080006]

12. Girmachew F. Identification on nontuberculosis mycobacteria from clinical specimens referred to national TB reference laboratory, Ethiopia. Presented at the 8th TB Research Advisory Committee (TRAC) Conference, 23 March 2013, Addis Ababa, Ethiopia. http://ehnri.go.
Day\%20Three/Feven\%20EDITED\%20Presentation.pdf (accessed 12 June 2013). 